\title{
Review: management of children with mental health problems by specialist teams in primary care improved some outcomes
}

Bower P, Garralda E, Kramer T, et al. The treatment of child and adolescent mental health problems in primary care: a systematic review. Fam Pract 2001 Aug;18:373-82.

\author{
QUESTION: What interventions are effective for treating mental health problems in \\ children and adolescents in primary care?
}

\section{Data sources}

Studies were identified by searching Medline, PsycINFO, CINAHL, EMBASE/Excerpta Medica, and the Cochrane Controlled Trials Register in August 1999; scanning the reference lists of relevant studies; and contacting 34 subject experts and authors of included studies.

\section{Study selection}

Studies were selected if they were randomised controlled trials (RCTs), controlled before and after studies, or before and after studies without controls and participants were $\leqslant 18$ years of age and were treated in primary care for mental health problems.

\section{Data extraction}

Data were extracted on design, study population, sample size, and results.

\section{Main results}

Educational studies involving the primary care team: 8 studies (1 RCT) were included. The RCT compared an introduction to the use of behaviour modification techniques (12 sessions) with a wait list control group in 14 health visitors who worked with 205 families. Groups did not differ for child behaviour. Studies involving treatment by the primary care team: 6 studies (3 RCTs) were included. 1 RCT compared 12 brief preschool interviews (20-30 $\mathrm{min}$ ) with no interviews over 5 years in a community sample of 246 non-distressed parents (and children). The intervention group showed improvement in some behaviour and relationship outcomes but no difference in school performance. Differences in neurotic symptoms persisted for up to 20-30 years. In 1 RCT of 90 children with night waking problems, no differences were seen at 12 weeks between groups receiving an advice booklet (with and without support from a researcher) and controls. 1 RCT $(n=25)$ showed that adolescents at risk of substance abuse who received an educational intervention by nurses (eg, pamphlets, motivational interview, and setting a contract of personal goals) reported greater reductions in alcohol use at 1 and 3 months than control participants. Management by specialists in primary care: 10 studies (3 RCTs) met the selection criteria. 1 RCT of 191 mothers with postpartum depression showed that treatment groups (counselling, cognitive behavioural therapy, and dynamic therapy) were better than routine primary care for maternal reports of infant relationship problems but not for mother-child interaction, cognitive development, or behavioural problems at 18 months. 1 RCT of 17 frequent attendees showed that the intervention group (group therapy by psychologist and general practitioner) had fewer family consultations than the control group (routine primary care). In 1 RCT of 260 preschool children with emotional and behavioural problems, 4 groups were compared: intensive health visiting, family therapy by social workers, mother and toddlers' groups run by social workers and health visitors, and no treatment. At 1 year, children in the family therapy group had lower cognitive development than children in the other 3 groups. At 3 years, groups did not differ.

\section{Conclusion}

In children and adolescents treated for mental health problems in primary care, evidence from a few randomised controlled trials suggests that preschool parental interviews can improve child behaviour and relationship outcomes and that management by specialists can reduce the number of consultations in frequent attendees.

Source of funding: Department of Health UK.

For correspondence: Dr P Bower, University of Manchester, Manchester, UK. pbower@

fsl.cpcr.man.ac.uk

\section{COMMENTARY}

Although it is clear that psychosocial problems in children and adolescents are frequently encountered in primary care, the systematic review by Bower et al exposes the surprising lack of adequate evidence-based knowledge on the effectiveness of interventions for this group.

Appropriately, the authors present a qualitative and not quantitative summary of the results. The studies included in the review reflect the British definition of primary health care. None the less, the most important message of this review is not diminished by the study selection methods. Through a detailed discussion of the methodological limitations of identified studies, Bower $e t$ al provide a template for future research in this area. For example, they highlight that many identified studies (a) failed to provide adequate baseline data on study participants, (b) did not include control groups or described randomisation poorly, (c) inadequately reported the actual intervention process, (d) rarely involved general practitioners, and (e) had inappropriate statistical analyses. Thus, a careful reading of this review should be mandatory for researchers preparing study proposals in this field.

For the clinician, it is encouraging to note that educational interventions for the primary care team, including collaborative teaching rounds, ${ }^{1}$ can be effective in increasing knowledge of common paediatric psychosocial issues. Given that behavioural modification therapy provided by specialists has had some documented success in reducing negative paediatric behaviours, teaching of these specific strategies to primary healthcare providers may be similarly effective.

This review by Bower $e t$ al, although limited in its conclusions, serves to identify research priorities. It may act as an effective stepping stone to a well designed randomised controlled trial of paediatric mental health interventions in primary care. Hema Patel, MD, MSc, FRCPC Montreal Children's Hospital Montreal, Québec, Canada

1 Fishman ME, Kessel W, Heppel DE, et al. Collaborative office rounds: continuing education in the psychosocial/developmental aspects of child health. Pediatrics 1997;99:E5. 